\title{
THE COHOMOLOGY OF CLASSIFYING SPACES OF $H$-SPACES
}

\author{
BY M. ROTHENBERG AND N. E. STEENROD ${ }^{1}$
}

Communicated by N. E. Steenrod, June 17, 1965

Let $G$ denote an associative $H$-space with unit (e.g. a topological group). We will show that the relations between $G$ and a classifying space $B_{G}$ are more readily displayed using a geometric analog of the resolutions of homological algebra. The analogy is quite sharp, the stages of the resolution, whose base is $B_{G}$, determine a filtration of $B_{G}$. The resulting spectral sequence for cohomology is independent of the choice of the resolution, it converges to $H^{*}\left(B_{G}\right)$, and its $E_{2^{-}}$ term is $\operatorname{Ext}_{H(G)}(R, R)$ ( $R=$ ground ring). We thus obtain spectral sequences of the Eilenberg-Moore type [5] in a simpler and more geometric manner.

1. Geometric resolutions. We shall restrict ourselves to the category of compactly generated spaces. Such a space is Hausdorff and each subset which meets every compact set in a closed set is itself closed (a $k$-space in the terminology of Kelley [3, p. 230]). Subspaces are usually required to be closed, and to be deformation retracts of neighborhoods.

Let $G$ be an associative $H$-space with unit e. A right $G$-action on a space $X$ will be a continuous map $X \times G \rightarrow X$ with $x e=x, x\left(g_{1} g_{2}\right)$ $=\left(x g_{1}\right) g_{2}$ for all $x \in X, g_{1}, g_{2} \in G$. A space $X$ with a right $G$-action will be called a $G$-space. A $G$-space $X$ and a sequence of $G$-invariant closed subspaces $X_{0} \subset X_{1} \subset \cdots \subset X_{n} \subset \cdots$ such that $X_{0} \neq \varnothing, X=\cup_{i=0}^{\infty} X_{i}$, and $X$ has the weak topology induced by $\left\{X_{i}\right\}$ will be called a filtered $G$-space.

1.1. Definition. (a) A filtered $G$-space $X$ is called acyclic if for some point $x_{0} \in X_{0}, X_{n}$ is contractible to $x_{0}$ in $X_{n+1}$ for every $n$.

(b) A filtered $G$-space $X$ is called free if, for each $n$, there exists a closed subspace $D_{n}\left(X_{n-1} \subset D_{n} \subset X_{n}\right)$ such that the action mapping $\left(D_{n}, X_{n-1}\right) \times G \rightarrow\left(X_{n}, X_{n-1}\right)$ is a relative homeomorphism.

(c) A filtered $G$-space $X$ is called a G-resolution if $X$ is both free and acyclic.

Under the restrictions we have imposed on subspaces, the acyclicity condition implies that $X$ is contractible.

1 This work was partially supported by the National Science Foundation under NSF grants GP 3936 and GP 2425. 
1.2. THEOREM. If $G$ is a topological group, any G-resolution $X$ is a principal $G$-bundle over $B_{G}=X / G$ with action $X \times G \rightarrow X$ as principal map.

When $G$ is a topological group, Milnor's construction [4], where $X_{n}$ is the join of $n+1$ copies of $G$, is a $G$-resolution. In the general case, the existence of a $G$-resolution is given by the Dold-Lashof construction [2].

There is also a comparison theorem. Let $G, G^{\prime}$ be $H$-spaces, $\Phi: G$ $\rightarrow G^{\prime}$ a morphism, $X, X^{\prime}$ filtered $G, G^{\prime}$-spaces. An extension $\Phi^{\prime}$ of $\Phi$ is a map $\Phi^{\prime}: X \rightarrow X^{\prime}$ with $\Phi^{\prime}\left(X_{n}\right) \subset X_{n}^{\prime}$ and $\Phi^{\prime}(x g)=\Phi^{\prime}(x) \Phi(g)$. If $\Phi^{\prime}, \Phi^{\prime \prime}$ are two extensions of $\Phi$, a homotopy $h$ will be a map $h: X \times I$ $\rightarrow X^{\prime}$ with $h_{0}=\Phi^{\prime}, h_{1}=\Phi^{\prime \prime}, h\left(X_{n} \times I\right) \subset X_{n+1}^{\prime}$, and $h(x g, t)=h(x, t) \Phi(g)$.

1.3. MAPping Theorem. If $\Phi: G \rightarrow G^{\prime}$ is a morphism, $X$ a free filtered $G$-space, $X^{\prime}$ an acyclic filtered $G^{\prime}$-space, then $\Phi$ has an extension $\Phi^{\prime}$ : $X \rightarrow X^{\prime}$. Furthermore, any two such extensions are homotopic.

Thus in particular, for any two resolutions $X, X^{\prime}$ of $G$ there exists an equivariant $\mu: X \rightarrow X^{\prime}$, unique up to equivariant homotopy.

We define the product of two filtered spaces $X, X^{\prime}$ to be the product space $X \times X^{\prime}$ filtered by $\left(X \times X^{\prime}\right)_{n}=\bigcup_{i=0}^{n} X_{i} \times X_{n-i}$.

1.4. TheOREM. If $X$ is a G-resolution and $X^{\prime}$ a $G^{\prime}$-resolution, then $X \times X^{\prime}$ is a $G \times G^{\prime}$-resolution.

2. The spectral sequence. When $X$ is a $G$-resolution, let $B=X / G$ denote the decomposition space by maximal orbits, let $p: X \rightarrow B$ be the projection and $B_{n}=p\left(X_{n}\right)$. If $R$ is a coefficient ring, the filtration $\left\{B_{n}\right\}$ of $B$ determines two spectral sequences, the homology spectral sequence $E_{*}(B, R)=\left\{E^{r}, d_{r}\right\}$ and the cohomology spectral sequence $E^{*}(B, R)=\left\{E_{r}, d^{r}\right\}$.

2.1. THEOREM. (a) The spectral sequences $E_{*}, E^{*}$ are functors from the category of $H$-spaces and continuous morphisms to the category of bigraded spectral sequences. (We regard all spectral sequences as beginning with $E^{2}, E_{2}$.)

(b) If the homology algebra $H(G)=H(G ; R)$ is $R$-free, then as a bigraded $R$-module

$$
E^{2} \cong \operatorname{Tor}^{H(G)}(R, R), \quad E_{2} \cong \operatorname{Ext}_{H(G)}(R, R) .
$$

(c) $E_{*} \Rightarrow H(B ; R)$. If $R$ is compact or $H(G)$ is free then $E^{*} \Rightarrow H^{*}(B ; R)$.

Proposition (a) follows from 1.3, (c) is true in any filtered space, and $(b)$ is proved using the Milnor-Dold-Lashof construction, in fact 
the $E^{1}$-term in this case is precisely the bar resolution of $R$ over the algebra $H(G)$.

In order to deepen these results to include products, we develop the theory of $X$-products for the spectral sequences of filtered spaces $X, Y$. These are natural transformations $\mu: E^{r}(X) \otimes E^{r}(Y)$ $\rightarrow E^{r}(X \times Y), \nu: E_{r}(X) \otimes E_{r}(Y) \rightarrow E_{r}(X \times Y)$ which behave nicely with respect to differentials. They are isomorphisms when $R$ is a field and $E_{1}(X)$ is of finite type.

The diagonal morphism $\Delta: G \rightarrow G \times G$ induces, by 2.1 (a), a mapping of the cohomology spectral sequences $\Delta^{*}: E_{r}\left(B_{G} \times B_{G}\right) \rightarrow E_{r}\left(B_{G}\right)$. Composing $\Delta^{*}$ with $\nu$ (where $X=Y=B_{G}$ ) gives the multiplication in $E_{r}$.

2.2. THEOREM. With respect to this multiplication, $E_{r}\left(B_{G}\right)$ is a commutative, associative, bigraded, differential algebra with unit. The multiplication on $E_{r+1}$ is induced by that on $E_{r}$. The multiplications commute with the convergence 2.1 (c). When $H(G)$ is $R$-free, the second isomorphism of 2.1(b) preserves products.

When $R$ is a field, the composition $\mu^{-1} \Delta_{*}$ defines a co-algebra structure in the homology spectral sequence having dual properties.

3. Co-algebra structure. We assume in this section that $R$ is a field and $H(G)$ is of finite type. When $G$ is commutative the multiplication $m: G \times G \rightarrow G$ is also a morphism. Then the composition $m_{*} \mu$ gives an algebra structure on $E_{*}$, and $\nu^{-1} m^{*}$ a co-algebra structure in $E^{*}$. Actually the same is true if $G$ is the loop space of an $H$-space. This yields

3.1. Theorem. If $G$ is commutative or the loop space of an $H$-space, then $E_{r}, E^{r}$ are bicommutative, biassociative, differential, bigraded Hopf algebras with $\left(E^{r}, d_{r}\right)$ the dual algebra to $\left(E_{r}, d^{r}\right)$. The Hopf algebra structure on $E_{2}=\operatorname{Ext}_{H(G)}(R, R)$ is the natural one arising from the Hopf algebra structure on $H(G)$. Moreover if $G$ is connected and $R$ is perfect, then $E_{r}$ is primitively generated on elements of bi-degree $(1, q),\left(2, q^{\prime}\right)$, and $d^{r}=0$ except for $r=p^{k}-1$ or $2 p^{k}-1$ where $p=$ Char $R$. If $G=\Omega(H)$, $H$ homotopy associative, then $E_{\infty} \approx H^{*}(B ; R)$ as an algebra.

Actually one can give an explicit description of $E_{r+1}$ in terms of $E_{r}$ and $d^{r}\left(x^{1, q}\right), d^{r}\left(x^{2, q^{\prime}}\right)$, where $x^{1, q}, x^{2, q^{\prime}}$ are primitive generators.

4. Applications. Moore pointed out [5] that his spectral sequence gives an easy proof of the theorem of Borel which states: If $H(G)$ is an exterior algebra with generators of odd dimensions and is $R$-free, then $H^{*}\left(B_{G}\right)$ is a polynomial algebra on corresponding generators of one higher dimension. Moore argues that a brief computation shows that 
the $E_{2}$-term, $\operatorname{Ext}_{H(G)}(R, R)$, is just such a polynomial algebra. Then all terms of $E_{2}$ of odd total degree are zero. Hence every $d^{r}=0$, so $E_{2}=E_{\infty}$. Since $E_{\infty}$ is a polynomial algebra, it is algebraically free; and therefore $H^{*}\left(B_{G}\right) \approx E_{\infty}$ as an algebra.

An Eilenberg-MacLane space of type $(\pi, n)$ can be realized by a commutative topological group $G$, and its $B_{G}$ is of type $(\pi, n+1)$. Consequently $H(\pi, n)$ and $H^{*}(\pi, n+1)$ are connected by a spectral sequence of Hopf algebras $E_{r}\left(B_{G}\right)$.

4.1. Theorem. If $G$ is of type $(\pi, n), \pi$ is a finitely generated abelian group, and $R=Z_{p}$ where $p$ is a prime, then the spectral sequence collapses

$$
\operatorname{Ext}_{H(G)}\left(Z_{p}, Z_{p}\right) \approx E_{2}=E_{\infty} \approx H^{*}\left(B_{G}\right) \text {. }
$$

This implies that $H^{*}\left(\pi, n ; Z_{p}\right)$ is a free commutative algebra for every $n$. In fact an algorithm is obtained for computing $H^{*}\left(\pi, n ; Z_{p}\right)$ as a primitively generated Hopf algebra over the algebra of reduced pth powers. These results confirm and amplify results of $H$. Cartan.

For another application, let $K$ be a compact, simply-connected Lie group, and let $G$ be the loop space of $K$. Using Bott's result [1] that $H(G ; Z)$ is torsion free, we obtain

\subsection{Theorem. (a) If $p>5$, the spectral sequence collapses}

$$
\operatorname{Ext}_{H(G)}\left(Z_{p}, Z_{p}\right) \approx E_{2}=E_{\infty} \approx H^{*}\left(K ; Z_{p}\right) \approx \Lambda\left(x_{1}, \cdots, x_{r}\right)
$$

where $x_{1}, \cdots, x_{r}$ are generators of the dimensions of the primitive invariants of $K$. In particular $K$ has no p-torsion, and $H^{*}\left(K ; Z_{p}\right)$ $\approx H^{*}(K ; Z) \otimes Z_{p}$.

(b) If $p=3$ or 5 , there is at most one nonzero differential, namely, $d^{2 p-1}$. Moreover $H^{*}\left(K ; Z_{p}\right)$ and $H_{*}\left(G ; Z_{p}\right)$ can be constructed explicitly from the Betti numbers of $K$ and the dimensions of the kernels of the maps $x \rightarrow x^{p}$ and $x \rightarrow x^{p^{2}}$ where $x \in H^{2}\left(G ; Z_{p}\right)$.

(c) For any $p>2$, we have $u^{p}=0$ for all $u \in \tilde{H}^{*}\left(K ; Z_{p}\right)$.

\section{BIBLIOGRAPHY}

1. R. Bott, The space of loops of a Lie group, Michigan Math. J. 5 (1958), 35-61.

2. A. Dold and R. Lashof, Principal quasifibrations and fibre homotopy equivalences, Illinois J. Math 3 (1959), 285-305.

3. J. L. Kelley, General topology, Van Nostrand, Princeton, N. J., 1955.

4. J. Milnor, Construction of universal bundles. II, Ann. of Math. 63 (1956), $430-436$.

5. J. C. Moore, Algebre homologique et cohomologie des espaces classifants, Séminaire H. Cartan, 1959-1960, Exposé 7.

University of Chicago and

Princeton University 